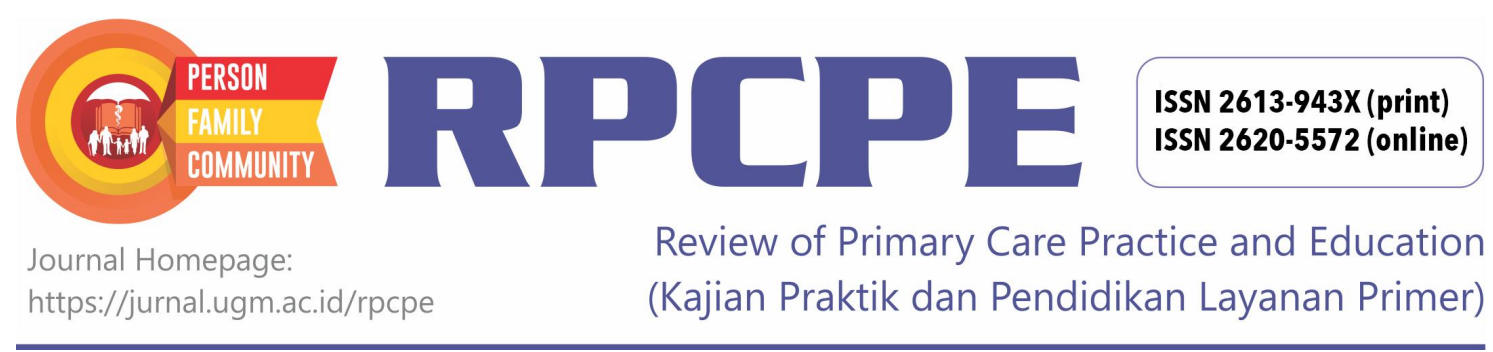

\title{
Pregnancy with Systemic Lupus Erythematosus (SLE)
}

\author{
Ika Hermawati ${ }^{1}$, Fitriana Murriya Ekawati ${ }^{2}$ \\ ${ }^{1}$ Puskesmas Nalumsari; Jawa Tengah; Indonesia \\ Training Participant of Primary Care Doctors Preceptors - Ministry of Health Republic of Indonesia 2017 \\ ${ }^{2}$ Department of Family and Community Medicine; Faculty of Medicine, Public Health, and Nursing; Universitas Gadjah Mada; \\ Indonesia
}

Corresponding Author:

Ika Hermawati: Primary Health Care Centre (Puskesmas) Nalumsari, Jl. Raya Jepara-Kudus Dusun 1, Pringtulis, Nalumsari, Kabupaten Jepara, Jawa Tengah - 59466, Indonesia

E-mail: vaniaaisya@yahoo.co.id

To cite this article:

Hermawati I, Ekawati FM. Pregnancy with systemic lupus erythematosus (SLE). Rev Prim Care Prac and Educ. 2019; 2(3): 111-114.

\section{CASE REPORT}

A 24-year-old female patient who was pregnant with a gestational age of 30 weeks came to attend an integrated prenatal check-up at Daren Village Hall at the invitation of the village midwife on April 14, 2017. During the examination, there were no complaints except a lack of appetite. The patient comes by the husband, but the husband cannot accompany him during the examination process because he will work. The patient has been married for two years, where the pregnancy at the time of this examination is the first pregnancy.

The patient's case history in 2015 shows that one month before marriage, the patient complained of severe bone pain accompanied by facial itching and one month of weight loss. The patient said that at that time he had examined himself at the Kudus General Hospital and was diagnosed with SLE. At that time, the patient received SLE therapy for two years. In early 2017, when the patient was declared pregnant, the patient was referred to Kariyadi Hospital and received therapy until the time this examination was carried out ( 7.5 months). The patient was managed by an internal medicine doctor from Kariyadi Hospital and was given $50 \mathrm{mg} \mathrm{1x1}$ of azathioprine, 1x1 of folic acid, $1 \times 2$ tab $(8 \mathrm{mg})$ of methylprednisolone, and vitamins from obstetricians.

The patient have been treated routinely at Kudus General Hospital, but after being pregnant the patient was referred to Kariyadi Hospital and received treatment for pregnancy and lupus in Kariyadi Hospital. So far, the patient have not received clear information about the disease, how it is treated, the impact on pregnancy, and how later if they will give birth whether they can be normal or not, they should give birth in Kariyadi Hospital or only midwives.

In psychology, the patient get full support from their families, husbands, and siblings regarding the treatment of their diseases. This can be seen from families who take turns to take the patient to control. In-laws also tell if they always provide various kinds of fruit for the patient to always be healthy as well as babies in their womb.

The patient has been married for two years, but has not had children and is only pregnant now. The patient does not work, only helps the husband guard the handphone counter shop. The husband works as an entrepreneur who has an handphone counter business and opens a motorcycle washing service at his home. The patient lives in his in-laws' house, where his parents-in-law only come once a month and stay in their house for approximately two weeks. Every day, the patient-in-law works selling pete and jengkol in Jakarta. When the in-laws are not at home, the patient sometimes spends time at the home of his parents who live near his residence (the village next to him). Two in-patient brothers and sisters residing only within four houses of the patient's residence.

From the results of the physical examination, no abnormalities were found, such as flares or joint pain. There is also no abnormality in the obstetric examination.

\section{Biological Diagnosis and Psychosocial Diagnosis}

The biological diagnosis is that a 30 -week pregnant woman with SLE is based on a physical examination conducted on the pregnancy that occurred as well as a history of lupus that has been in the treatment period for two years until now.

The psychosocial diagnosis of this patient, namely a 24year-old woman who is pregnant for 30 weeks with SLE, is afraid that her illness will interfere with pregnancy and go down to her child and the place of treatment that isfar enough.

\section{FORMULATION OF THE PROBLEM}

The problem, in this case, is not about medical because lupus has received adequate therapy from experts in 
Kariyadi Hospital. However, the problems are (1) How should monitoring be done so that the patient can undergo a safe pregnancy even if he is suffering from lupus?; (2) How can the baby growth be optimal and be able to undergo a safe delivery?; (3) Can this patient give birth in primary care considering there are no complications?; (4) What is the care of the patient and their babies after childbirth?; (5) How can the patient reduce negative feelings and thoughts about their illness and be able to return to active activities other than homework?

\section{DISCUSSION}

Systemic Lupus Erythematosus or better known as SLE is one of the autoimmune rheumatic diseases that require special attention both in recognizing the clinical appearance of the disease and its management. As a doctor in primary care, general practitioners should understand that this disease has a thousand faces ${ }^{1,2}$. The patient does not present with the typical symptoms that he has SLE, but it can be just itching, redness of the face and joint pain, where the doctor may give a wrong diagnosis with allergies or arthritis. If there are patients with symptoms that lead to SLE, patients should then be tested for SLE antibody (ANA test) and if the results are positive, they can be followed up with SLE therapy/referral ${ }^{3}$.

Both men and women can be attacked by this disease, but predominance is more prominent in women of reproductive $\mathrm{age}^{4}$. It also concerns all races even though more is seen in women in Asia, or those who are black in America. SLE disease is so dynamic that it often makes diagnosis difficult when medical professionals are faced with an incomplete display of symptoms or complaints. Early recognition of the possibility of someone affected by this disease is very important, given the death rate can occur quickly related to disease activity in the first years. Meanwhile, further complications, especially in the cardiovascular system and the disruption of various organ functions as the natural course of the disease progresses, also make a major contribution to the morbidity and mortality of patients with SLE or often referred to as people with lupus/orang dengan lupus (ODAPUS).

Diverse manifestations, often not realized by medical professionals who face these the patient. Not infrequently, for days, weeks to months or even years the patient are diagnosed based on the dominant manifestations seen as anemia, glomerulonephritis, acneiform dermatitis, and so on $^{5}$. Manifestations that can occur with a long time span. Delay in establishing the diagnosis will affect the level of management success and survival of patients with SLE. Complete management will determine good output. The benefits felt by the patient will also be more evident. Various complications that can be prevented certainly increase the survival of ODAPUS, avoid the use of unnecessary drugs or even reduce the cost of treatment and utilization of health facilities. This SLE treatment pillar should be carried out simultaneously and continuously so that treatment goals are achieved. Efforts to monitor diseases ranging from general practitioners at the periphery to the level of consultant doctors, especially rheumatologists.

\section{Pregnancy with SLE}

Pregnancy in mothers with Systemic Lupus Erythematosus (SLE) disease is closely related to the level of pain and death of the mother and fetus. The risk of death of pregnant women suffering from SLE has an impact 20 times higher due to complications caused by preeclampsia, thrombosis, infections and blood disorders ${ }^{6}$.

The problem that worsens the situation during pregnancy is the occurrence of disease flares, especially if the activity of SLE disease is high before becoming pregnant. Flare in pregnancy is reported to be between 13 to $68 \%$ of pregnant SLE sufferers compared to non-pregnant women.

The prognosis of pregnant women who suffer from SLE is determined at conception, if conception occurs during the remission, the prognosis will be better. According to Kwok, Tam, Zhu, Leung, and Li, if SLE than six months before conception there is a history of nephritis and active SLE disease with a SLEDAI score of 4 or more will be at risk of adversely affecting the fetus ${ }^{7}$. Strengthened by Roy, Das, and Datta that SLE sufferers who have experienced a remission period more than six months before pregnancy have a $25 \%$ risk of exacerbation during pregnancy compared to if the SLE remission period before pregnancy is SLE than six months then the risk of SLE exacerbations at the time get pregnant to $50 \%$ with the impact of a bad pregnancy $^{8}$.

This shows that pregnancy in people with SLE is very much determined by the activity of the disease, the conception that occurs during remission has a good pregnancy impact compared to before achieving remission. With a disease that is stable or suffers from flares that are relatively rare or only a little in pregnancy will give birth to a healthy baby. Therefore, education and monitoring of pregnant women during pregnancy is very important. There are several things that need to be done by pregnant women with SLE during their pregnancy so that they are healthy and give birth to healthy baby, namely: (1) Diligently do pregnancy checks (ANC), to monitor the condition of the mother and fetus; (2) Conduct periodic laboratory checks as recommended by the doctor; (3) Take medicines and vitamins given by the doctor; (4) Consuming nutritious balanced foods.

In this case, even though the patient has no complications related to the disease, labor is still recommended at advanced health facilities in order to get more supervision and awareness of complications that may occur during labor, such as preeclampsia and bleeding. Babies born to mothers with lupus and none complications will get treatment as well as the management of a healthy baby, while babies born with complications such as IUGR, premature and other abnormalities will get management according to the case ${ }^{9}$.

\section{The Role of Family Physicians in Primary Care}

Family physicians in primary care can play a more comprehensive role in dealing with SLE patients. The first and second pillars of lupus management are Education/ Counseling and Rehabilitation. In this case both will be 
discussed.

\section{(1) Education/Counseling}

(a) Education/Counseling

Basically, SLE the patient need correct information and support from the surrounding in order to be able to live independently. Need to be explained about the course of the disease and its complexity. Patients need knowledge of physical activity problems, reducing or preventing recurrence including protecting the skin from sun exposure (ultra violet) by wearing sunscreen, umbrellas or hats; do exercise regularly. Patients must pay attention if they have an infection. Need to regulate the diet so as not to be overweight, osteoporosis or dyslipidemia. Information is needed to monitor various organ functions, both related to disease activities or due to the use of drugs. Educational items in SLE the patient are: 1) A description of what lupus is and what causes it; 2) Type of SLE disease and temperament of each type; 3) Physical related problems: the usefulness of exercise especially those related to steroid use, osteoporosis, rest, overcoming infections, contraceptive use; 4) Introduction to the problem of psychological aspects: how to understand SLE the patient themselves, overcome fatigue, emotional stress, psychological trauma, problems related to family or workplace and work itself, overcoming pain; 5) Use of drugs includes type, dosage, duration of administration, and so on. Whether or not a mineral and vitamin supplementation is needed; 6) Where the patient can obtain information about SLE, are there support groups, foundations that are engaged in correcting SLE and so on.

(b) Biopsychosocial Approach of SLE Management Regarding the biopsychosocial approach in SLE management, each SLE patient needs to be analyzed for neuropsychological and social problems. Based on research data at the RSCM found cognitive impairment of $86.49 \%$. The proof is done using a more thorough examination tool such as A TRAIL, TRAIL B, or Pegboard. This shows the magnitude of neuropsychiatric disorders that are hidden in SLE because, in reality, the disorder does not exceed $20 \%$. The presence of psychological stigmata in the patient's family still needs further verification. But the presence of physical and cognitive disorders in SLE the patient can have a detrimental effect on the patient in their social environment both at work and home.

Family education is directed at reducing the impact of the psychological stigmata due to the presence of families with SLE, providing information on the need for excessive family support. This is so that the patient with SLE can be understood by their families and are able to be independent in their daily lives.

(c) Continuity of Care in Primary Care

Family physicians in primary care will continue to see patients with SLE, at least when these patients will renew their referral with a specialist. But actually more than that. The doctor met this patient probably from the time he was a teenager to old age. Some of the following educational tips and practices can be done on these patients: 1) SLE is a hereditary disease. As many as $44 \%$ of SLE cases are hereditary cases that can be reduced ${ }^{1,2,3}$. The doctor can continue to observe the possibility of this case appearing in children with SLE or educate the hereditary possibility of this case in survivors of SLE; 2) Provision of vitamin D. Vitamin $\mathrm{D}$ is given to prevent bone loss; 3) Vaccination. SLE patients are very susceptible to infections, therefore vaccination is important. Live-vaccine vaccines are usually contraindicated in patients on corticosteroid therapy such as SLE. However, attenuated vaccines (do not contain live germs) can be given with an approach in each case. Usually recommended are pneumococcal and influenza vaccines. This vaccine is given when the patient is in a controlled condition, including HPV vaccination for the prevention of cervical cancer in SLE patients ${ }^{1,2,3}$; 4) Attention to reproductive functions. If pregnant, patients with SLE have a higher risk for preeclampsia-eclampsia. This possibility increases equally in patients with lupus nephritis with predisposing factors namely hypertension and anti-phospholipid syndrome (APS). Management of SLE before, during pregnancy and after delivery is very important. Things that must be considered include: a) If SLE sufferers want to get pregnant it is recommended that at least after 6 months the disease activity is controlled or in a state of total remission. In lupus nephritis the period lasts up to 12 months in total remission. This can reduce lupus recurrence during pregnancy; b) Medical: Corticosteroid dose is as small as possible, i.e. not to exceed $7.5 \mathrm{mg} /$ day prednisone or equivalent. DMARDs or other medicines should be given with caution. Pay attention to recommendations before giving these medications; c) Provision of low-dose aspirin to reduce the risk of preeclampsia during pregnancy. Provision of aspirin is certainly done in collaboration with obstetricians; d) Because the patient has a high risk of preeclampsia, labor should be performed in the hospital; e) Prompts to use contraception: contraception is recommended to provide adequate pause time, so that the patient can recover well before the next pregnancy, while reducing the risk of preeclampsia and eclampsia in subsequent pregnancies. Various contraceptives can be given. Contraception using a condom or IUD is a safe choice. Contraception with hormonal methods can be done more carefully considering the recurrence of symptoms of SLE patients. Non-combined hormonal contraception (only containing progesterone is recommended if the patient is not suitable to use combined hormonal contraception).

\section{(2) Rehabilitation Program}

There are various modalities that can be given to the patient with SLE depending on the intent and purpose of this program. One important thing is understanding the decline in muscle mass by up to $30 \%$ if the patient with SLE are left in immobility for more than 2 weeks. Besides that, a decrease in muscle strength will occur around $1-5 \%$ per day in immobility conditions. Various exercises are needed to maintain joint stability. Physical modalities such as providing heat or cold are needed to reduce pain, eliminate stiffness or muscle spasm. Likewise other modalities such as Transcutaneous Electrical Nerve Stimulation (TENS) 
provide considerable benefits in the patient with muscle pain or stiffness. The fertility of SLE sufferers is the same as the female population, not SLE. Some studies have lupus recurrence during pregnancy but are generally mild, but if the pregnancy occurs while nephritis is still active, then 50$60 \%$ exacerbations, while if lupus nephritis is in a remission state 3-6 months before conception only $7-10 \%$ experience recurrence.

\section{REFERENCES}

1. Gergianaki I, Bertsias G. Systemic lupus erythematosus in primary care: an update and practical messages for the general practitioner. Frontiers in Medicine. 2018 May 29;5:161.

2. Mok CC. Systemic lupus erythematosus: What should family physicians know in 2018. Hong Kong Medical Journal. 2018 Oct 1;24:501-11.

3. VU LAM NC, Ghetu MV, Bieniek ML. Systemic lupus erythematosus: Primary care approach to diagnosis and management. American Family Physician. 2016 Aug 15;94(4).

4. Data and Information Center. Lupus situation in Indonesia. Jakarta: Ministry of Health Republic of Indonesia. 2017. Available from: http:/ www.depkes.go.id/resources/download/pusdatin/infodatin/InfodatinLupus-2017.pdf.

5. Indonesia Rheumatology Association. Diagnosis and management of lupus erythematosus. Jakarta: Indonesia Rheumatology Association. 2011.

6. Varghese S, Crocker I, Bruce IN, Tower C. Systemic lupus erythematosus, regulatory $\mathrm{T}$ cells and pregnancy. Expert Review of Clinical Immunology.2011 Sep 1;7(5):635-48.

7. Kwok LW, Tam LS, Zhu TY, Leung YY, Li EK. Predictors of maternal and fetal outcomes in pregnancies of patients with systemic lupus erythematosus. Lupus. 2011 Jul;20(8):829-36.

8. Roy JS, Das PP, Datta A. SLE in pregnancy. BSMMU Journal 2010;3(1):54-9. Available from: https://pdfs.semanticscholar. org/6f87/9807b65d8f655b8aa33814638b72ad513052.pdf.

9. Knight CL, Nelson-Piercy C. Management of systemic lupus erythematosus during pregnancy: Challenges and solutions. Open Accessrheumatology: Research and Reviews. 2017;9:37. 